\title{
LECCIONES APRENDIDAS Y TAREAS PENDIENTES EN LA ACTIVIDAD REPOBLADORA Y VIVERÍSTICA
}

\author{
Jesús Pemán García \\ Departamento de Producción Vegetal y Ciencia Forestal-AGROTECNIO Center. Universidad de Lleida. \\ Avda. Rovira Roure 191. 25199-LLEIDA (España)
}

\section{Resumen}

En estos últimos años ha habido nuevas aportaciones al conocimiento en el ámbito del viverismo y de la repoblación forestal. Se ha profundizado en el conocimiento de los factores que influyen en el arraigo, es decir, el genotipo, las condiciones ambientales, las interacciones entre las plantas y el fenotipo de la planta cultivada en vivero, así como en los procesos fisiológicos que lo condicionan. Se realiza un breve análisis de los nuevos avances y de las tareas que quedan pendientes. También se analiza la estructura del proceso repoblador en su conjunto y la necesidad de establecer futuras directrices o protocolos que garanticen un correcto diseño, la participación social, su control de calidad y su seguimiento y evaluación en el tiempo.

Palabras clave: Arraigo, Genotipo, Calidad de planta, Facilitación, Condiciones ambientales, Proyecto

\section{LOS NÚMEROS DE LA ACTIVIDAD REPOBLADORA Y VIVERÍSTICA}

No es fácil, en la actualidad, valorar en el tiempo de una forma cuantitativa la actividad repobladora y viverística por la falta de series completas y homogéneas que la describan. Sirva como ejemplo la discrepancia que existe sobre la superficie repoblada en tres informes: i) Evaluación de los recursos mundiales de la FAO en 2010 (FAO, 2010), ii) los Anuarios de Estadística Forestal del Ministerio de Agricultura, Alimentación y Medio Ambiente (MAGRAMA) y iii) Informe elaborado en 2010 por la Sociedad Española de Ciencias Forestales sobre la Situación de los bosques y del sector forestal en España (S.E.C.F., 2010) (Tabla 1).

Los esfuerzos realizados para tratar de reconstruir estas series (PEMÁN Y VADELL, 2009) permiten indicar que la actividad repobladora desarrollada en España desde el último cuarto del siglo XIX hasta 2010, supera los 5,35 millones de hectáreas (Mha) (Figura1a). Difiere notablemente esta cifra

\begin{tabular}{|l|l|c|c|c|}
\hline Referencia & Variable & $\mathbf{1 9 9 0 - 2 0 0 0}$ & $\mathbf{2 0 0 1 - 2 0 0 5}$ & $\mathbf{2 0 0 6 - 2 0 1 0}$ \\
\hline FAO & Área de bosque plantado (miles ha) & 467 & 45 & 130 \\
\hline SECF & Superficie repoblada (miles ha) ${ }^{1}$ & $1.006^{2}$ & $222^{3}$ & \\
\hline MAGRAMA & Superficie repoblada (miles ha) ${ }^{1}$ & $814^{4}$ & 183 & 268 \\
\hline
\end{tabular}

Tabla 1. Valores de la superficie repoblada en España entre 1990 y 2010, según diferentes informes. Abreviaturas: FAO: Organización de las Naciones Unidas para la Agricultura y Alimentación, SECF: Sociedad Española de Ciencias Forestales, MAGRAMA: Ministerio de Agricultura, Alimentación y Medio Ambiente. Fuentes: (FAO, 2010; S.E.C.F, 2010; MAGRAMA, 2014). ${ }^{\prime}:$ engloba superficie de repoblaciones productoras, protectoras y forestación de tierras agrarias. ${ }^{2}$ : abarca el periodo 1994-2000, ${ }^{3}$ : no hay datos en 2003 y 2004, ${ }^{4}$ : abarca el periodo 1992-2000 
de la superficie que la FAO, en su informe de 2010 (FRA2010), considera como superficie de bosque plantado en España, dado que lo estima en 2,68 Mha (FAO, 2010). Según la FRA2010 la superficie de bosques plantados en el mundo asciende a 264 Mha y en Europa, sin la Federación Rusa, a 52,3 Mha. Según los valores recogidos en la FRA2010, la superficie de bosque plantado en España representa el 5\% de la europea y el $1 \%$ de la mundial. Si se refiere la extensión del bosque plantado en España a la superficie forestal total del país (27,7 Mha) y a la superficie forestal arbolada (18,2 Mha) (FAO, 2010), la extensión del bosque plantado representa un $9,7 \%$ y un $14,7 \%$, respectivamente. Concentrando el análisis de esta actividad a partir de 1994, se puede comprobar como la mayor parte de las repoblaciones realizadas se enmarcarían en el Programa de forestación de tierras agrarias iniciado ese año como consecuencia de la reforma de la Política Agrícola Comunitaria en 1992 (Figura 1b). La superficie total forestada con cargo a este programa entre 1994-2010 sería de 726.405 ha que representa el $67 \%$ de toda la superficie repoblada en ese periodo (1.083.164 ha). Desagregando este valor según el objetivo de la repoblación, el $73 \%$ de la superficie repoblada ha tenido un objetivo preferente protector, mientras que el $27 \%$ restante lo ha tenido productor (MAGRAMA). Los recursos económicos con los que se ha realizado esta actividad repobladora han sido fundamentalmente públicos (Figura 1d). Estas inversiones mostraban una tendencia alcista en el periodo 2005-2010 (Figura 1c), que en la actualidad habrán retrocedido con motivo de la crisis económica, probablemente a niveles inferiores a los del año 2005, es decir, a 70 millones de euros. Este nivel de inversión representaba entre el 5 y el $10 \%$ de la inversión pública del sector forestal y menos del 0,2\% del total de la inversión pública (ASEMFO). Estas cifras contrastan con el peso de la inversión pública en la restauración ambiental, que en 2010, sólo en trabajos de integración paisajística, la inversión de Adif (Administrador de infraestructuras ferroviarias) fue de 74 millones de euros.

En cuanto a la actividad viverística es difícil obtener una estimación del número de viveros productores de planta forestal por lo irregular de la serie de datos, su posible falta de homogenei-
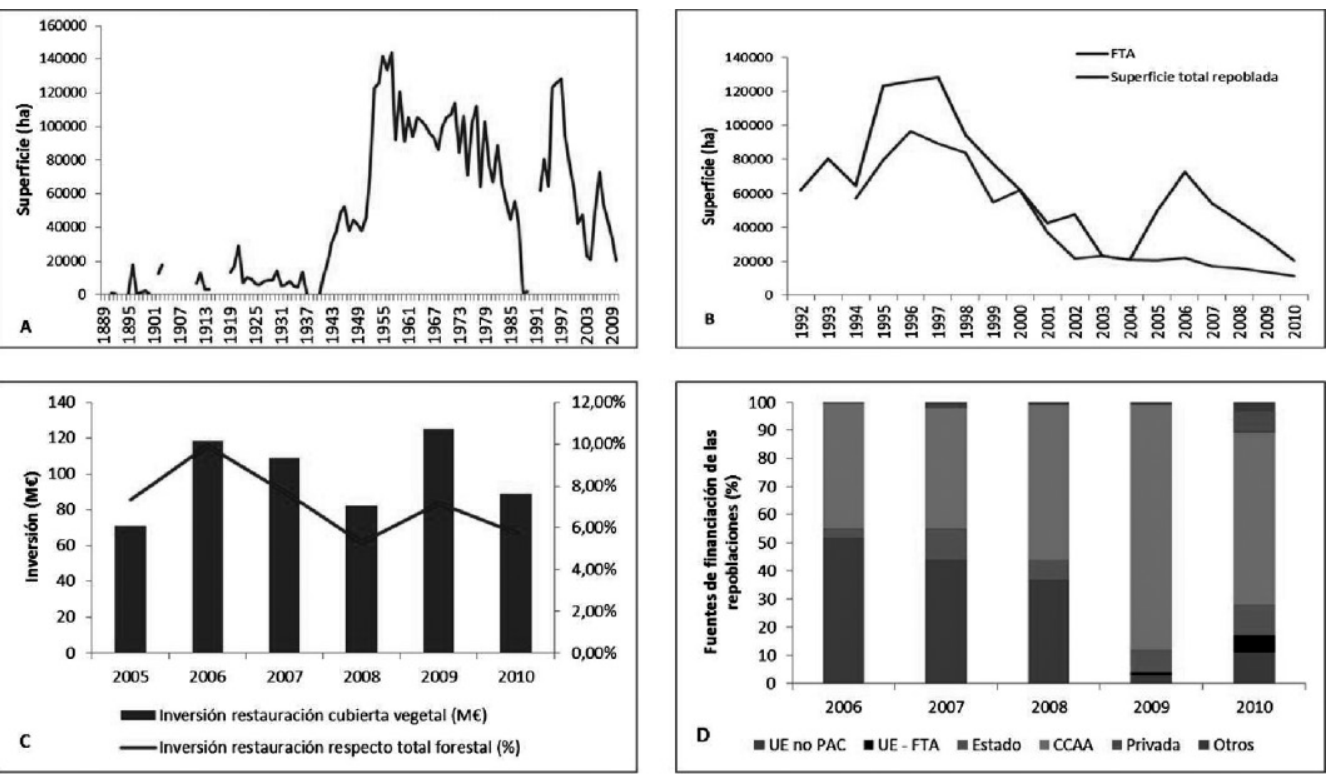

Figura 1. Evolución de la superficie total anual repoblada en España entre 1891 y 2010 (A), la superficie total repoblada y la superficie forestada por el programa de forestación de tierras agrarias (FTA) entre 1994 y 2010 (B), la inversión en restauración de la cubierta vegetal y su porcentaje respecto a la inversión forestal total entre 2005 y $2010(C)$, las fuentes de financiación de la actividad repobladora (D). Fuentes: (PEMÁN Y VADELL, 2009; ASEMFO; MAGRAMA) 
dad, por las oscilaciones que se registran entre años y por haber desaparecido esta variable en la estadística forestal desde el año 2009. Se podría considerar, como cifra consolidada en la década anterior, la existencia de 400 viveros forestales, aunque los dedicados a la producción de planta forestal podrían ser muchos menos (Figura 2a). La producción de planta forestal ha registrado en estos últimos años sus valores mínimos, oscilando entre 20 y 40 millones de plantas, muy lejos de los 1.200 millones del año 1957 o de los 150 millones de la década de los noventa del siglo pasado (Figura 2b). A la vista de las limitaciones que presenta la estadística forestal en el ámbito de las repoblaciones y los viveros forestales se puede decir que la estadística forestal es una lección desaprendida. Las series actuales son incompletas, se recurren a estimaciones de algunas variables, cambian las variables recogidas en el tiempo y no se recogen algunas de las variables que pueden ayudar a caracterizar esta actividad. Especialmente son deficientes las estadísticas que hacen referencia al ámbito viverístico, aspecto que sorprende enormemente por ser un sector muy regulado que debe presentar una declaración anual de cultivo.

La actividad repobladora ha ido acompañada de una notable de labor de investigación y divulgación que ha permitido generar un cuerpo de conocimiento sobre la misma en cada periodo de tiempo. Si hasta 1940 los artículos publicados en las revistas técnicas españolas sobre repoblaciones se acercaban a 400, las publicaciones hasta 1990 superaban las 700. A partir de 1990, si atendemos solo a las publicaciones realizadas en revistas científicas o congresos internacionales o a las realizadas en congresos nacionales o reuniones de los grupos de trabajo organizadas por la Sociedad Española de Ciencias Forestales, el número de publicaciones es muy notable (Figura 3), lo que ha permi-
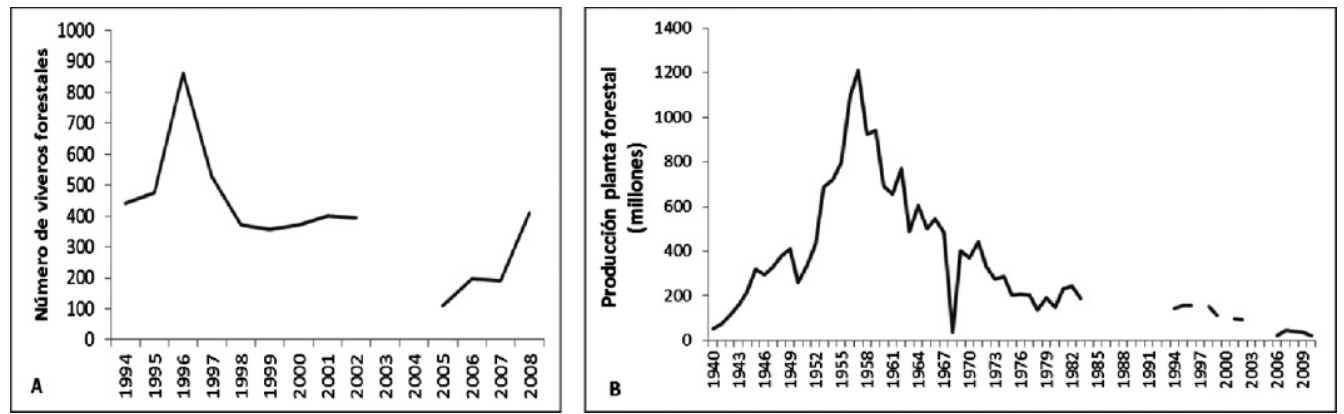

Figura 2. Evolución del número de viveros forestales en España en el periodo 1994-2008 (A) y de la producción de planta forestal en España durante el periodo 1940-2010 (B). Fuentes: (PEMÁN Y VADELL, 2009; ASEMFO; MAGRAMA)

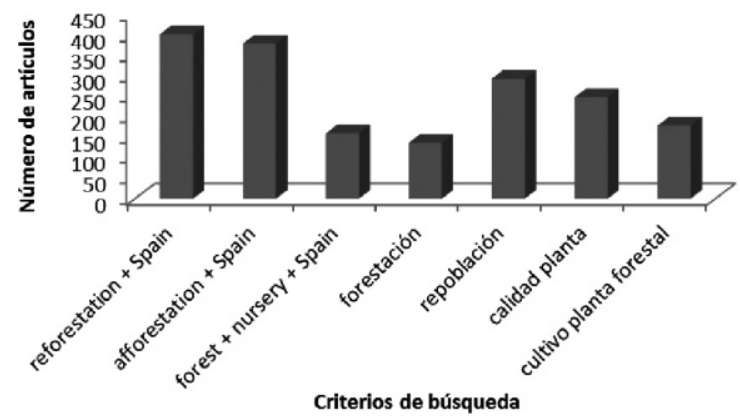

Figura 3. Número de publicaciones, a partir de 1990, según los criterios de búsqueda en la base de datos CAB Abstracts (recoge los tres primeros criterios de la búsqueda) y en el buscador de publicaciones de la Sociedad Española de Ciencias Forestales (recoge el resto) 
tido disponer de un amplio cuerpo de conocimientos que permite mejorar las técnicas a aplicar.

\section{Objetivo}

El objetivo de esta ponencia es poner de relieve los conocimientos y buenas prácticas adquiridas durante los últimos años en el ámbito de las repoblaciones y viveros forestales, así como evidenciar las tareas que quedan pendientes y que deberían ser afrontadas en los próximos años. Este análisis se va realizar en dos ámbitos: i) Factores que condicionan el arraigo o establecimiento, ii) Proceso restaurador desde su fase de proyecto.

\section{FACTORES QUE CONDICIONAN EL ESTABLECIMIENTO O ARRAIGO EN EL MONTE}

El éxito del establecimiento de una repoblación viene determinado por la tasa de supervivencia y el ritmo de crecimiento de las plantas introducidas, que condicionarán la constitución de la masa forestal deseada y, por ende, los fines que de ella se demandan. En el ambiente mediterráneo, donde la supervivencia dependerá fundamentalmente del grado con que la planta es capaz de superar el estrés hídrico, el desarrollo del sistema radicular en el monte determinará la capacidad de captación del agua y nutrientes y por tanto, sus posibilidades de supervivencia y crecimiento. La supervivencia vendrá determinada, por tanto, por la capacidad de desarrollo radicular para satisfacer la mayor o menor demanda de recursos según las condiciones de la estación. Los modelos conceptuales de BURDETT (1990) y MARGOLIS \& BRAND (1990) que evidencian los procesos fisiológicos que interactúan en el arraigo han sido recientemente modificados por VILLAR-SALVADOR et al. (2012), que han incorporado al modelo algunos aspectos que definen el fenotipo de la planta cultivada en vivero como es la concentración de nitrógeno o el tamaño de la planta (Figura 4). La aceptación de estos modelos permite visualizar la interrelación entre los distintos factores y procesos que pueden influir en el desarrollo radicular y como consecuencia en el arraigo, aunque alguno de ellos todavía está sometido a profundo debate.

Se considera que el establecimiento de la planta después de la plantación está condicionado principalmente por (BURDETT, 1990): i) el genotipo, ii) las condiciones ambientales, iii) las interacciones entre plantas y iv) el fenotipo de la planta cultivada en vivero.

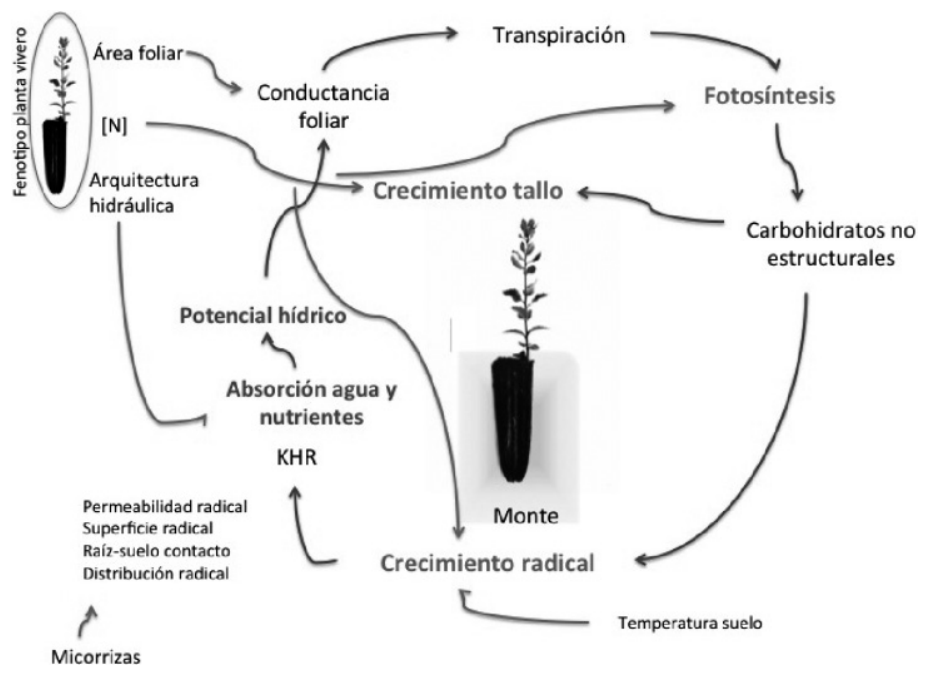

Figura 4. Esquema teórico de los procesos fisiológicos que relacionan el crecimiento radical con la fotosíntesis y el fenotipo de la planta producida en vivero elaborado a partir de los modelos de BURDETT (1990), MARGOLIS \& BRAND (1990) y VILLAR-SALVADOR et al. (2012). KHR: Conductancia hidráulica radicular, [N]: concentración de nitrógeno 


\section{El genotipo}

La influencia del origen de las semillas en el comportamiento posterior de las plantas (fenología, adaptación, crecimiento, calidad de los productos, etc.) en las especies forestales con amplias áreas de distribución fue puesto en evidencia hace más de 200 años y los primeros ensayos de procedencias en Europa fueron realizados por Philippe André de Vilmorin en 1823 sobre Pinus sylvestris. El inicio de los estudios sistemáticos sobre procedencias en Europa se remonta a los realizados en 1929 sobre Picea abies y Pinus sylvestris (ElORRIETA, 1946). A pesar de estas evidencias, los repobladores en España tardaron en aceptar que las diferencias que podían encontrarse entre los individuos de una misma especie podían tener, además de un componente ambiental, un componente genético (PARDOS, 1988).

Hoy en día, podemos decir que, en el ámbito de las repoblaciones forestales, la elección de la calidad genética del material forestal de reproducción es una de las decisiones estratégicas del proyecto de repoblación. No obstante, quedarían como temas pendientes trasladar la importancia de esta elección a dos tipos de actuaciones: la restauración ambiental en el ámbito de la obra civil (transporte, hidráulica, minería, etc.) y las plantaciones truferas. En el primero de los casos se precisa, como paso previo, que la restauración ambiental se enfoque desde el ámbito de la restauración ecológica (VALLADARES et al., 2011) y no desde la jardinería, con objeto de poder integrar adecuadamente el sistema creado en la restauración con el circundante a la misma. En este sentido, hay que tener en cuenta la importante función que desempeñan las infraestructuras lineales como verdaderos corredores que dependiendo de la bondad de las soluciones adoptadas pueden convertirse en conectores o en barreras que contribuyan a la fragmentación de los hábitats y a la contaminación genética de poblaciones o especies existentes. Para evitarlo, debe generalizarse para las especies que no tienen regulada su comercialización el empleo de las denominadas Regiones de Identificación y Utilización (RIUs) como referencia de la procedencia de los materiales. En el ámbito de la truficultura, con más de 10.000 ha repobladas en toda la península (REYNA et al., 2013), es necesario hacer cumplir la normativa de comercialización. Aunque también en este ámbito es evidente la influencia que un determina- do origen produce en el comportamiento de la planta en monte (REYNA Y COLINAS, 2007), la planta utilizada en las plantaciones no solía contar con el documento de acompañamiento y etiqueta que acreditase su procedencia, aunque normalmente provenía de fuentes semilleras locales. En este ámbito, dado que las especies generalmente utilizadas tienen reguladas su comercialización, a excepción de Corylus avellana, hay que exigir el cumplimiento de la normativa.

El número de especies forestales que tiene regulada su comercialización y, por tanto, su calidad genética, tanto a nivel europeo, estatal como autonómico son: 48 para el conjunto de la Unión Europea, 76 en el Estado español, 80 en Castilla y León y 116 en la Comunidad Valenciana (Tabla 2).

El Registro Nacional de Materiales de Base para las especies que tienen regulada su comercialización por el Estado cuenta en la actualidad con un número importante de unidades de admisión para las diferentes categorías del material forestal de reproducción (Tabla 3), lo que permite al repoblador cierta capacidad de elección, que disminuye a medida que es más alta la calidad genética exigida al material.

A la vista de estos datos, y al margen de los comentado sobre la necesidad de determinar la procedencia de los materiales en las plantaciones truferas o en las actuaciones de restauración ambiental, es necesario realizar un esfuerzo dirigido a: i) Disponer de materiales de las categorías cualificada o controlada para un mayor número de especies. En este sentido, hay que continuar con los trabajos realizados, años atrás, para la selección y posterior mejora de materiales para las especies con una posible demanda continuada en el tiempo, como ocurre con las especies productoras de madera de calidad (MONTERO et al., 2002). Sirva como ejemplo, que de las dieciocho especies recogidas en esta publicación solo se dispone de material de la categoría cualificada en tres de ellas. ii) Seguir avanzando en el conocimiento del comportamiento de los materiales en diferentes estaciones o ambientes para poder establecer las oportunas recomendaciones de uso de los mismos para el repoblador. iii) Conocer las procedencias o genotipos que presentan una mayor eficiencia en el uso del agua, como alternativa al contexto de cambio. iv) Disponer del material vegetal que elija el repoblador. Para ello hay conocer con suficien- 


\begin{tabular}{|c|c|c|}
\hline Legislación & Tipo especie & Número especies \\
\hline \multirow{3}{*}{$\begin{array}{l}\text { Estatal } \\
(\text { RD.289/2003) }\end{array}$} & \multirow[t]{2}{*}{ Arbórea } & $\begin{array}{l}\text { Autóctonas: } 47 \\
\text { Abies alba, A.pinsapo, Acer platanoides, A.pseudoplatanus, Alnus glutinosa, } \\
\text { Betula pendula, B.pubescens, Carpinus betulus, Castanea sativa, Fagus } \\
\text { sylvatica, Fraxinus angustifolia, F. excelsior, Ilex aquifolium, Juglans regia, } \\
\text { J.thurifera, Olea europea, Phoenix canariensis, Pinus uncinata, Pistacia } \\
\text { atlantica, Pinus canariensis, } P \text {. halepensis, } P \text {. nigra, P. pinaster,P. pinea, } \\
\text { Psylvestris, Populus alba, P. nigra, P. tremula, Prunus avium, Quercus } \\
\text { canariensis, Q. faginea, Q. pyrenaica, Q. cerris, Q. ilex, Q. petraea, Q. pubescens, } \\
Q . \text { robur, Q. suber, Sorbus aria, S. aucuparia, Tamarix gallica, Taxus baccata, } \\
\text { Tetraclinis articulata, Tilia cordata, T. platyphyllos, Ulmus minor, } U . \text { glabra }\end{array}$ \\
\hline & & $\begin{array}{l}\text { Alóctonas: } 23 \\
\text { Abies cephalonica, A.grandis, Alnus incana, Híbridos artificiales de Castanea } \\
\text { sativa, Cedrus atlantica, C.libani, Juglans spp. e híbridos artificiales entre estas } \\
\text { especies, Larix decidua, L x eurolepis, L. kaempferi, L.sibirica, Picea abies, } \\
\text { P. sitchensis, Pinus brutia, P. cembra, P. contorta, P. leucodermis P. radiata, } \\
\text { híbridos artificiales de Populus, Pseudostuga menziesii, Quercus rubra, } \\
\text { Robinia pseudoacacia }\end{array}$ \\
\hline & Arbustiva & $\begin{array}{l}6 \\
\text { Arbutus canariensis, A. unedo, Juniperus communis, J. oxycedrus, J. phoenicea, } \\
\text { Quercus coccifera }\end{array}$ \\
\hline $\begin{array}{l}\text { Castilla y León } \\
\text { (RD.54/2007) }\end{array}$ & Arbórea & $\begin{array}{l}4 \\
\text { Acer campestre, A. monspessulanum, Celtis australis, Sorbus domestica }\end{array}$ \\
\hline \multirow{4}{*}{$\begin{array}{l}\text { Comunidad } \\
\text { Valenciana } \\
\text { (D.15/2006) }\end{array}$} & Arbórea & $\begin{array}{l}11 \\
\text { Acer campestre, A. monspessulanum, A. opalus subsp. granatense, Amelanchier } \\
\text { ovalis, Celtis australis, Fraxinus ornus, Salix alba, S. atrocinerea, S. fragilis, } \\
\text { Sorbus domestica, S. torminalis, }\end{array}$ \\
\hline & Arbustiva & $\begin{array}{l}19 \\
\text { Atriplex halimus, Buxus sempervirens, Chamaerops humilis, Crataegus monogyna, } \\
\text { Phillyrea angustifolia, Ph. latifolia, Pistacia lentiscus, P. terebinthus, Prunus } \\
\text { mahaleb, P. spinosa, Rhamnus alaternus, R. lycioides, Salix elaeagnos, Salix } \\
\text { purpurea, Tamarix africana, T. boveana, T. canariensis, Viburnum lantana, V. tinus }\end{array}$ \\
\hline & Matorral & $\begin{array}{l}8 \\
\text { Erica arborea, E. multiflora, Juniperus sabina, Myrtus communis, Osyris lanceolata, } \\
\text { Periploca laevigata ssp. angustifolia, Salsola genistoides, S. oppositifolia }\end{array}$ \\
\hline & Herbácea & $\begin{array}{l}2 \\
\text { Lygeum spartum, Stipa tenacissima }\end{array}$ \\
\hline
\end{tabular}

Tabla 2. Especies que tienen regulada la comercialización y uso de sus materiales forestales de reproducción según la normativa estatal o autonómica actualmente vigente

te antelación la demanda real de materiales que van a ser utilizados en las labores de repoblación. Esto obliga a que los proyectos de repoblación tengan una duración temporal que permita, si es el caso, la recolección y producción del material forestal que va ser utilizado.

\section{Las condiciones ambientales}

A priori, sobre este factor el repoblador tiene menor capacidad de actuación, aunque puede influir, para mejorar el establecimiento, por medio de la preparación del suelo, la elección correcta de la época de plantación, el empleo de tubos invernadero o el aumento de la disponibilidad hídrica, que son los aspectos sobre los que se va realizar una revisión del estado del conocimiento.

\section{Preparación del suelo}

La variedad de técnicas disponibles en la actualidad ha propiciado la realización de ensayos experimentales que han tratado de valorar su influencia sobre la supervivencia y el crecimiento de las plantas o su efecto sobre las propiedades físicas del suelo que han sido recopilados en trabajos anteriores (SERRADA et al., 2005). Las mejores 


\begin{tabular}{|c|c|c|}
\hline Categoría & Número de taxones & $\begin{array}{l}\text { Material de Base. Naturaleza y } \\
\text { número de unidades de admisión }\end{array}$ \\
\hline Identificada & 63 & Fuentes semilleras: 7.215 \\
\hline Seleccionada & $\begin{array}{l}17 \\
\text { Abies alba, Castanea sativa, Fagus sylvatica, híbridos } \\
\text { artificiales Castanea sativa, Pinus canariensis, } P \text {. halepensis, } \\
\text { P. nigra salzmanii, P. pinaster, P. halepensis, P. nigra } \\
\text { salzmanii, P. pinaster, P. pinea, P. radiata, P. sylvestris, } \\
\text { P. uncinata, Quercus petraea, Q. robur, Q. rubra, Q. suber }\end{array}$ & Rodales: 338 \\
\hline Cualificada & $\begin{array}{l}15 \\
\text { Acer pseudoplatanus, híbridos artificiales Castanea sativa, } \\
\text { Juglans regia, J. nigra, híbridos Juglans spp., Pinus } \\
\text { halepensis, } P \text {. nigra nigra, P. nigra salzmanii, P. pinaster, } \\
\text { P. radiata, P. sylvestris, } P \text {. uncinata, Populus } x \\
\text { euramericana, Prunus avium, Pseudotsuga menziesii }\end{array}$ & $\begin{array}{l}\text { Huertos semilleros: } 24 \\
\text { Clones: } 55 \\
\text { Progenitores familia: } 31\end{array}$ \\
\hline Controlada & $\begin{array}{l}9 \\
\text { Híbridos artificiales de Castanea sativa, Pinus pinaster, } \\
\text { P. radiata, Populus spp. }\end{array}$ & $\begin{array}{l}\text { Huertos semilleros: } 2 \\
\text { Clones: } 41 \\
\text { Progenitores familia: } 4\end{array}$ \\
\hline
\end{tabular}

Tabla 3. Composición del Registro Nacional de Materiales de Base. Fuente: (NICOLAS E IGLESIAS, 2012) y elaboración propia mediante consulta en abril de 2013

tasas de supervivencia y crecimiento se han registrado en las preparaciones intensas, como los ahoyados con retroexcavadora, banquetas con microcuencas, los subsolados o las terrazas mecanizadas, justificado por el mayor volumen de suelo removido, la reducción de la resistencia al crecimiento radical para alcanzar los horizontes profundos, la mejora en la infiltración y en la disponibilidad del agua en el suelo. Es por ello, que se debe renunciar en los ambientes mediterráneos a las preparaciones del suelo de carácter manual o poco intensas, que en su día fueron propuestas como alternativas viables (VILAGROSA et al., 1997). Los estudios sobre la influencia de los diferentes procedimientos sobre las propiedades físicas o químicas del suelo ha permitido conocer que las preparaciones mecanizadas frente a las manuales disminuyen la resistencia a la penetración de las raíces, mejoran la fertilidad y la capacidad de almacenamiento de agua en el suelo si van acompañadas de enmiendas orgánicas (QUEREJETA et al., 2001) o han registrado que la humedad del suelo esta correlacionada directamente con el tamaño del poro, el contenido en arcilla y la cantidad de carbonato cálcico equivalente e inversamente con el tamaño de la partícula (GUTIÉRREZ-RAVÉ, 2007). Como recomendación, se puede indicar que para esperar un efecto favorable de la preparación del suelo en los ambientes mediterráneos habrá que garantizar, al menos: i) un correcta elección del procedimiento, basada en un estudio detallado del suelo, entre otros aspectos, ii) un correcto dimensionamiento de las estructuras, para garantizar su capacidad de recolección de agua (MONGIL Y MARTÍNEZ DE AZAGRA, 2006; MoNGIL et al., 2012) y iii) un control de su ejecución. Hay que tener muy presente, además, las posibles interacciones que se pueden producir entre la calidad de planta, la época de plantación y la intensidad de la preparación, puestas de manifiesto por varios autores (South et al., 2001; PALACIOS et al., 2009).

\section{Época de plantación}

La interpretación de la época de la plantación o siembra como un factor trascendente en el arraigo (PAlACios et al., 2009) y su falta de consideración en numerosos ensayos experimentales que han tratado de valorar la influencia de distintos factores en la supervivencia y crecimiento de la planta después de la plantación, ha planteado la necesidad de estudiar su influencia sobre el éxito de la repoblación (RAdOGlou et al., 2003; PALACIOS et al., 2009), sobre la actividad radical, como responsable directa del establecimiento de la planta (CORCHERO et $a l ., 2002$; VALLE et $a l ., 2005$ ) o sobre el nivel de estrés de la planta, a través de estudios de proteómica (ARIZA et al., 2008). Dado que la plantación exige que la planta esté en parada vegetativa, la época de plantación varía entre mediados de otoño y finales de invierno en estaciones de media o baja montaña, desplazándose a lo largo de la primavera 
en la alta montaña. Dentro de este intervalo de tiempo, plantar temprano, mediados de otoño, o plantar tarde, finales del invierno, puede influir en el arraigo. En un ensayo sobre Quercus frainetto y Castanea sativa se demostró que las plantaciones tempranas presentaron durante su primer año crecimiento un mayor potencial hídrico al mediodía, una mayor área foliar y una mayor supervivencia que las plantaciones más tardías (RADOGLOU et al., 2003), asimismo, sobre Quercus ilex se comprobó que las plantaciones tempranas combinadas con preparaciones intensas registraron las mejores tasas de crecimiento en altura y diámetro, mostrándose como el factor más influyente en la supervivencia de la plantación para esta especie (PALACIOS et al., 2009). Sobre Pinus halepensis, Pinus pinea y Quercus ilex se han realizado estudios sobre cinética del crecimiento aéreo y radical y la influencia que en este último tiene la temperatura del suelo, según la fecha de plantación. Han mostrado la conveniencia de las plantaciones tempranas, a final de octubre, al permitir el desarrollo radical antes de que la temperatura del suelo alcance los valores mínimos para el crecimiento durante el invierno, estimados en $5^{\circ} \mathrm{C}$ para los pinos y $10^{\circ} \mathrm{C}$ para la encina, y al registrar el escaso desarrollo radical con el que afrontan las especies el periodo seco en las plantaciones tardías (CORCHERO et al., 2002; VALLE et $a l ., 2005)$. Los estudios realizados sobre los perfiles proteicos producidos en plantas de
Pinus halepensis como consecuencia de la época de plantación, han identificado una variación cuantitativa y cualitativa en proteínas, posiblemente indicadores de estrés, en el mes de abril frente al mes de febrero, lo que indicaría una conveniencia de las plantaciones tempranas (ARIZA et al., 2008). Cabe, por tanto, recomendar en el ámbito mediterráneo las plantaciones tempranas de mediados de otoño, siempre después de que se haya producido la recarga de agua del suelo después del periodo seco, por ser una de las decisiones que contribuyen más a disponer de un desarrollo radical de garantías para afrontar el periodo estival. La elección correcta de la época de plantación debe extenderse, también, al ámbito de la restauración ambiental donde los plazos de ejecución del proyecto de obra suele imponer unas fechas de plantación o siembra no idóneas para el arraigo de la vegetación.

\section{Empleo de protectores}

El uso de protectores se ha generalizado desde los programas de forestación de tierras agrarias con el objetivo de mejorar la supervivencia y crecimiento de las plantas ubicadas en su interior, aunque sin conocer realmente los efectos que los diferentes modelos podían tener sobre las mismas. Bajo la denominación de protectores se encuentran productos de naturaleza muy variada que exigen la determinación de algunas de sus características para poder predecir el resultado final de su uso (Tabla 4).

\begin{tabular}{|l|l|l|l|l|}
\hline Tipo de material & Finalidad & $\begin{array}{l}\text { Variables } \\
\text { ambientales } \\
\text { que modifica }\end{array}$ & Parámetros a definir \\
\hline Protectores en espiral & Protección física & - & Altura, modelo \\
\hline Protectores de malla & $\begin{array}{l}\text { Mallas } \\
\text { cinegética }\end{array}$ & Protección física & - & $\begin{array}{l}\text { Material, luz de la malla, } \\
\text { gramaje (plásticos) y altura }\end{array}$ \\
\cline { 2 - 5 } & $\begin{array}{l}\text { Mallas de } \\
\text { sombreo }\end{array}$ & $\begin{array}{l}\text { i) Protección física } \\
\text { ii) Creación de un } \\
\text { ambiente favorable } \\
\text { para el crecimiento }\end{array}$ & Luz, temperatura & $\begin{array}{l}\text { Luz de la malla, gramaje } \\
\text { y altura }\end{array}$ \\
\hline Tubos invernadero & $\begin{array}{l}\text { i) Protección física } \\
\text { ii) Creación de un } \\
\text { ambiente favorable } \\
\text { para el crecimiento }\end{array}$ & $\begin{array}{l}\text { Luz, temperatura, } \\
\text { humedad, viento, } \\
\text { concentración de } \mathrm{CO}_{2}\end{array}$ & $\begin{array}{l}\text { Color, altura, ventilación, } \\
\text { estabilidad }\end{array}$ \\
\hline Tubo malla & $\begin{array}{l}\text { i) Protección física } \\
\text { ii) Creación de un } \\
\text { ambiente favorable } \\
\text { para el crecimiento }\end{array}$ & $\begin{array}{l}\text { Luz, temperatura, } \\
\text { humedad, viento }\end{array}$ & Color, altura \\
\hline
\end{tabular}

Tabla 4. Tipos de protectores, características y finalidad de uso 
La modificación que producen de las condiciones microclimáticas del aire confinado en su interior y su incidencia en el crecimiento y supervivencia en ambientes mediterráneos ha suscitado numerosos ensayos cuyos resultados han sido recogidos en trabajos anteriores (SERRADA et al., 2005), siendo, todavía, objeto de estudio. Considerado resuelto el problema de sobrecalentamiento en el interior del tubo con la elección del sistema correcto de ventilación, los trabajos en la actualidad se centran en la caracterización del ambiente lumínico y su incidencia sobre el crecimiento de la planta, según su grado de tolerancia a la sombra de la especie (DEL CAMPO et al., 2008; PuÉrtolas et al., 2010; VÁzQUEZ et $a l$., 2013). El sombreo que produce el tubo se ha mostrado clave en simulaciones en modelos 3D (PEMÁn et $a l ., 2010)$ y en ensayos de campo (Puértolas et al., 2010) mostrando que, en especies tolerantes a la sombra, el sombreo aumenta la eficiencia en el uso del agua tanto en primavera como en verano, una reducción del estrés hídrico y una reducción de la temperatura foliar. La aparición en el mercado de modelos de tubos malla, presentan una alternativa muy interesante a las mallas de sombreo, por su mayor estabilidad, y puede ser recomendado para las especies que no precisan o toleran un sombreo pero si de cierta protección física contra herbívoros y otros agentes físicos como el viento (PEMÁn et al., 2011). A la vista de estos estudios debe evitarse la generalización del uso de tubos invernadero en ambientes mediterráneos. La utilización del tubo, por tanto, debe ser justificada y la elección del modelo constituye una decisión estratégica del proyecto, por la variación que producen en el ambiente lumínico y en la temperatura del aire confinado en su interior. En su elección el repoblador debe asegurarse de la idoneidad del grado de sombreo que produce el tubo con la tolerancia de la especie así como de la efectividad del tipo de ventilación para evitar un sobrecalentamiento excesivo. Debe continuarse la caracterización de los nuevos materiales que van apareciendo, así como la determinación del ambiente lumínico más apropiado según los grados de tolerancia de las especies de cara a futuros nuevos diseños.

\section{Aumento de la disponibilidad hídrica}

Aumentar la disponibilidad de agua para la planta en su fase de establecimiento y manteni- miento en zonas áridas o semiáridas ha sido un cuidado cultural que se practicó entre las medidas de acompañamiento del programa de forestación de tierras agrarias bien mediante riego directo, microrriego (SÁNCHEZ et al., 2004; MARTínEZ DE AZAGRA Y DEL Río, 2012) o con el uso de hidrogeles (HÜTTERMANN et al., 1999; CLEMENTE et al., 2004; Del CAMPo et al., 2008; BAI et al., 2010; DeL CAMPO et al., 2011). Las diferentes técnicas de microrriego pueden ser alternativas a tener en cuenta en el contexto de zonas áridas o semiáridas en restauraciones singulares aunque no debe plantearse su posibilidad de uso de forma general (MarTínez de AZAGRa y Del Río, 2012). Sobre el uso de hidrogeles, que se ha generalizado en algunas zonas de nuestra geografía, no hay evidencias claras que permitan extraer conclusiones debido a la diversidad de resultados obtenidos por el tipo de producto utilizado, las dosis empleadas o las características del suelo donde se ha realizado el ensayo, por lo que deberán seguirse los estudios antes de extender su uso a las repoblaciones en general.

\section{Las interacciones entre plantas}

De las relaciones de carácter interespecífico entre las plantas en el proceso de repoblación se ha prestado especial atención a dos de ellas, la facilitación y la competencia (CALLAWAY \& WALKER, 1997). Aunque la valoración de estas relaciones no siempre es fácil por la presencia simultánea de varias interacciones y por la influencia que en las mismas tienen el resto de los elementos bióticos y abióticos del ecosistema, se ha profundizado en su conocimiento con la intención de sacar conclusiones para el ámbito de las repoblaciones y de la restauración en general. La competencia interespecífica en un ecosistema se manifiesta por el espacio físico, aéreo y radical, y por los recursos como la luz, el agua, los nutrientes y el $\mathrm{CO}_{2}$ (CAllaway \& WalKer, 1997). A excepción de este último, el resto de los recursos pueden ser más o menos limitantes en las diferentes estaciones, por lo que las plantas deberán compartirlos; limitándose, así, su capacidad de crecimiento. En las estaciones mediterráneas la competencia se suele establecer sobre el agua, el recurso más limitante en este medio, hecho que ha originado gran parte de las especializaciones entre las plantas. La composición específica de la vegetación competidora está influenciada clara- 
mente por la historia de uso y de gestión previa del terreno (GACHET et al., 2007) y la intensidad de la competición según la calidad de la estación, es un aspecto, hoy en día, sometido a debate (REES, 2013). Las técnicas de control de la vegetación en las repoblaciones forestales han sido ampliamente descritas en todo el ámbito europeo (WILloughBy et al., 2009), poniendo en evidencia la dualidad competidora-facilitadora que muchas especies presentan según la disponibilidad de recursos. La facilitación abarca la interacción beneficiosa que una especie produce sobre otra al aportarle sombreo, un aumento en el contenido de nutrientes, un aumento en la humedad y aireación del suelo, protección contra herbívoros, un aumento de visitas de polinizadores y de diseminadores o la eliminación de competidores (BROOKER et al., 2008). Hoy en día, la importancia de la facilitación, en el desarrollo de las comunidades vegetales sometidas a fuertes estreses abióticos y bióticos, atrae a numerosos ecólogos y ha sido puesta en evidencia en diferentes biomas (BROOKER et al., 2008). En esta línea se vienen realizado ensayos en el ámbito mediterráneo para poder aprovechar los mecanismos de la facilitación en el proceso de restauración con especies arbóreas al amparo de especies arbustivas nodrizas, generalmente leguminosas, que faciliten su supervivencia y crecimiento (GómEZAPARICIO et al., 2004; CASTRO et al., 2004; TORROBA et al., 2013). Aunque los resultados muestran una clara influencia positiva, debe profundizarse en su conocimiento mediante estudios temporales de mayor duración y sometidos a gradientes ambientales para poder valorar los signos y la intensidad de las interacciones. El conocimiento de las mismas y su variación en el tiempo puede ser estratégico en los procesos de restauración pasiva, más que para actuaciones de restauración activa, al identificar las especies que pueden ejercer el papel de nodriza para respetarlas y promocionarlas con el objetivo de que se constituyan en promotoras de los futuros procesos de regeneración natural. Una de las primeras aplicaciones a escala paisaje del mecanismo de la facilitación han sido los modelos de restauración forestal clásicos con diferentes formaciones arbóreas (RUIZ DE LA TORRE, 1993; LUGO, 1997). En nuestro contexto, está siendo objeto de numerosos estudios el modelo de facilitación Pinus-
Quercus con objeto de determinar su grado de éxito y la idoneidad del uso de los pinos como especies pioneras en el proceso restaurador (PAusas et al., 2004; Gómez-Aparicio et al., 2009). La validez y pertinencia de estos modelos de restauración forestal ha sido analizada en varios estudios siendo objeto de un intenso debate (MAestre et al., 2003; PAUSAS et al., 2004; GONZÁLEZ-MorENo et al., 2011). Se ha observado, en algunos trabajos, que bajo la cubierta del pinar los diseminadores como el arrendajo (Garrulus glandarius) han favorecido la progresiva entrada de rebrotadoras (GómEZ, 2003; PONS Y PAUSAS, 2007), siendo este proceso clave para la recuperación de las zonas quemadas (PUERTAPIÑERO et $a l .$, 2011), mientras que en otros muchos casos la progresiva naturalización del pinar no se ha conseguido, presentando estas masas una reducida resiliencia y una marcada vulnerabilidad a las perturbaciones (ANDRÉS Y OJEDA, 2002). Estudios recientes han mostrado como la diversificación espontánea de las masas de pináceas artificiales se asocia a variables relacionadas con la estructura de la masa, la distancia a fuentes semilleras, la posición en la ladera o el grado de fragmentación de la masa (GómEZAPARICIO et al., 2009; GONZÁLEZ-MORENO et al., 2011). En los casos en que la diversificación de los ecosistemas no se consigue de manera natural, se ha propuesto desde distintos ámbitos el desarrollo de prácticas de gestión para favorecer este proceso, como pueden ser la ejecución de pequeñas plantaciones de diversificación en masas monoespecíficas que tendrían el papel de actuar como de focos de dispersión para estas especies (CARreras, 2006; PrÉvosto et al., 2011).

\section{El fenotipo}

La interacción de un genotipo determinado, cuya influencia en el establecimiento de la planta ya ha sido tratada independientemente, con las diferentes condiciones ambientales de cultivo en los viveros conduce a la obtención de diferentes fenotipos, sobre los que el repoblador tiene que hacer la elección correcta según las características de la estación donde van a ser empleados. La contribución que el fenotipo de la planta producida en vivero tiene sobre el arraigo es evidente en la medida que condiciona arquitectura hidráulica, la capacidad fotosintética y la capacidad de des- 
arrollo radical de la planta en las condiciones de monte, como ha quedado puesto en evidencia en el modelo teórico expuesto en la Figura 4. Se ha avanzado en el conocimiento de los protocolos de producción de planta para numerosas especies forestales y en la definición de unas ventanas de calidad o rangos de variación para algunos atributos, según la terminología de RITCHIE (1984), que pueden expresar o predecir la respuesta de la planta en el monte como ha quedado ampliamente recogido en algunas publicaciones monográficas (CORTINA et al., 2006; PeMÁn et al., 2012) (Tabla 5). El objetivo de establecer estas ventanas de calidad es servir de orientación para el usuario final y para el viverista, al permitirle estandarizar el cultivo de la especie. A pesar de estos avances, el debate sigue abierto tanto en lo que hace referencia a los estándares de calidad de la planta como en lo que hace relación las técnicas de producción. Bajo la sugerente pregunta ¿por qué las plantas sobreviven?, GROSSNICKLE (2012) analiza la influencia que los diferentes atributos pueden tener en la respuesta de la planta en monte. Para este autor, los atributos morfológicos como la altura, el diámetro en el cuello de la raíz, el peso seco radical o el ratio peso seco aéreo/peso seco radical (PSA/PSR), permiten describir la arquitectura hidráulica de la planta y pueden ayudar a definir cuál es la más apropiada para cada especie y estación. Un mayor diámetro en el cuello de la raíz y una mayor biomasa radicular pueden ser buenos predictores, con carácter general, de la supervivencia mientras que la altura del tallo y el ratio PSA/PSR precisan ajustarse a las características de la estación.

Por otro lado, Villar-Salvador et al. (2012) proponen con carácter general como planta de referencia una planta grande, equilibrada entre su parte aérea y radical, y con un buen estado nutricional para favorecer el rápido desarrollo radical en la época húmeda y así hacer frente con más probabilidades de éxito a la época seca. El modelo propuesto por los mismos incide en un debate arraigado en el ámbito viverístico forestal español sobre si la planta más pequeña es más adecuada al ámbito mediterráneo que la planta grande pero, atendiendo a lo dicho anteriormente, este tipo de planta será eficaz en la medida que puede desarrollar el sistema radical que precisa antes de la época estival

\begin{tabular}{|c|c|c|}
\hline $\begin{array}{l}\text { Tipo de } \\
\text { atributo }\end{array}$ & Variables & Especies \\
\hline \multirow[t]{2}{*}{ Morfológico } & altura, diámetro cuello raíz & $\begin{array}{l}\text { RD. 289/2003: Abies pinsapo, Pinus uncinata, P. sylvestris, } \\
\text { P. nigra, P. pinaster, P. pinea, P. halepensis, Quercus ilex, } \\
\text { Q. faginea, Q. pirenaica, Q. suber. } \\
\text { Norma francesa: Acer platanoides, A. pseudoplatanus, Alnus } \\
\text { glutinosa, Betula pendula, B. pubescens, Fagus sylvatica, } \\
\text { Fraxinus excelsior, Larix sp., Prunus avium, Pseudotsuga } \\
\text { menziessi, Quercus petraea, Q. robur, Tilia spp. } \\
\text { Otras especies: Apollonias barbujana, Arbutus canariensis, } \\
\text { Persea indica, Phoenix canariensis, Juglans spp. } \\
\end{array}$ \\
\hline & $\begin{array}{l}\text { altura, diámetro cuello raíz, } \\
\text { peso seco aéreo, peso seco } \\
\text { radicular, ratio PSA/PSR, } \\
\text { esbeltez, índice de Dickson }\end{array}$ & $\begin{array}{l}\text { Abies pinsapo, Pistacia lentiscus, P. terebinthus, Arbutus } \\
\text { unedo, Pinus canariensis, P. pinea, P. sylvestris, } \\
\text { Quercus suber, Ceratonia siliqua, Olea europea var. } \\
\text { sylvestris, Chamaerops humilis, Crategus monogyna, } \\
\text { Juniperus phoenicea, Myrtus communis, Nerium oleander }\end{array}$ \\
\hline Fisiológico & $\begin{array}{l}\mathrm{N}, \mathrm{P}, \mathrm{K}, \mathrm{Ca} \text { y } \mathrm{Mg} \text { foliar, } \mathrm{N}, \\
\mathrm{P}, \mathrm{K} \text { raíz, carbohidratos } \\
\text { reserva, N parte aérea }\end{array}$ & $\begin{array}{l}\text { Abies pinsapo, Pistacia lentiscus, P. terebinthus, Arbutus } \\
\text { unedo, Pinus canariensis, } P \text { pinea, } P \text {. sylvestris, } \\
\text { Quercus suber, Ceratonia siliqua, Olea europea var. sylvestris, } \\
\text { Juniperus thurifera, Pinus nigra, Quercus coccifera, Q. } \\
\text { faginea, Q. ilex, Retama sp., Pinus halepensis. }\end{array}$ \\
\hline Respuesta & $\begin{array}{l}\text { Potencial regeneración } \\
\text { radical }\end{array}$ & $\begin{array}{l}\text { Abies pinsapo, Pistacia lentiscus, P. terebinthus, Ceratonia } \\
\text { siliqua, Olea europea var. sylvestris, Pinus halepensis }\end{array}$ \\
\hline
\end{tabular}

Tabla 5. Especies que tienen definidos intervalos o ventanas de calidad para diferentes atributos morfológicos, fisiológicos o de respuesta (PEMÁN et al., 2012) 
(GrossniCKLE, 2012). Dada la importancia que en el establecimiento tiene que la planta desarrolle un sistema radical que le permita captar los recursos que precisa para su supervivencia y crecimiento en las condiciones de estación es preciso profundizar en el estudio de atributos que permitan describir con mayor precisión la funcionalidad de dicho sistema. El peso o el volumen radical, la fibrosidad, la relación tallo, raíces nuevas formadas, la forma o el potencial de regeneración radical son alguno de los parámetros en los que se debe profundizar (DAVIS \& JACOBS, 2005; HAASE, 2011; GROSSNICKLE, 2012). Los atributos de estado de carácter fisiológico y de respuesta como el estado nutricional, la tolerancia al frío, el contenido en carbohidratos de reserva o el potencial de regeneración radical, pueden ser buenos estimadores, también, de la capacidad de desarrollo radical y por tanto de la supervivencia en monte aunque con un efecto más efímero sobre la planta después de la plantación (GROSSNICKLE, 2012). Está pendiente la determinación de los requerimientos de calidad de planta para las actuaciones de restauración ambiental. El tipo de planta demandada en este tipo de obras es la de planta grande que exige, por tanto, un mayor tiempo en el vivero y precisa de una determinación correcta de los tipos de envase a utilizar, el sustrato de cultivo, en el que juega un papel importante no solo sus propiedades físicas o químicas sino su estabilidad, o los programas de riego y fertilización.

En cuanto a las técnicas de producción se ha avanzado mucho en relación a contenedores, sustratos, riego o fertilización, existiendo recomendaciones generales para un gran número de especies. En el ámbito de la fertilización un paso importe ha sido la sustitución del uso de fertilizantes de liberación lenta, que imposibilitaban el conocimiento real del cuándo y cuánto del aporte de nutrientes, por la fertirrigación. Sin embargo, quedan aspectos en los que se deben profundizar, sobre todo en el ámbito de la aplicación de técnicas de endurecimiento hídrico y nutricional para hacer frente a los ambientes más desfavorables.

Es necesario fomentar la integración de viveristas, repobladores e investigadores con objeto de poner en común protocolos de cultivo, estándares de calidad, programas de control integrado de cultivo o para el diseño de estudios en campo multi-ambiente para poder conocer la diversidad de respuestas de las plantas para los diferentes estándares de calidad (CORTINA et al., 2006).

\section{El proceso repoblador}

A pesar de la gran superficie repoblada en España, a día de hoy, no se dispone de herramientas que permitan realizar una evaluación global de la actividad repobladora realizada tanto en su fase de diseño, ejecución y seguimiento.

El diseño de la actividad repobladora con fines protectores, en los tiempos actuales, no ha respondido a ninguna directriz general, ni se ha ajustado a ningún protocolo, reglamento o pliego de condiciones, ajustándose únicamente a lo que cada técnico ha creído conveniente desarrollar en cada proyecto. En la exposición de motivos del Reglamento e Instrucciones de repoblación forestal de 1933 se justifica: ...la existencia de una disposición oficial que recoja con el debido detalle lo anteriormente expuesto [los estudios que han de realizarse, los documentos que deben tener todo proyecto, etc.] es de tal necesidad que sin ella todo plan de repoblación forestal, cualquiera que sea su amplitud carecería de una idea directriz y de las indispensables garantías de un buen planteamiento y una acertada ejecución. Merece la pena recordar que en este mismo reglamento se estructuraba el diseño de las actuaciones en: $i$ ) Memorias de reconocimiento general, ii) Estudio de las secciones, iii) Propuestas anuales y iv) Revisión de los proyectos en ejecución, donde se describirá la marcha de los trabajos, su ajuste al proyecto, los resultados logrados, el coste de los trabajos y su comparación con lo presupuestado. Hoy en día, la rapidez con que se diseñan y ejecutan los proyectos imposibilita, en muchos casos, realizar el análisis con el detalle que precisan sus condicionantes, por ejemplo el suelo, ni garantizan disponer de algunas de las alternativas elegidas, por ejemplo la categoría del material forestal de reproducción. En cuanto a la ejecución y seguimiento se dispone de escasa información sobre la realidad de la misma y las posibles alteraciones que haya sufrido el proyecto inicial, así como de sus resultados. En este sentido, se está desaprovechando una magnífica oportunidad de mejorar el estado del conocimiento a la vista de los resultados obtenidos con 
las técnicas empleadas sobre los diferentes ambientes y calidades de estación.

Desde diferentes ámbitos se están haciendo esfuerzos para contar con un protocolo normalizado a seguir para el proyecto restaurador en ecosistemas forestales que facilite su posible certificación (HERNÁNDEZ, 2013) o para diseñar pliegos de condiciones particulares para determinados aspectos como el manejo del material forestal de reproducción (IGLESIAS et al., 2012).

La necesidad de disponer de unos protocolos o instrucciones para el proceso repoblador se hace aún más necesaria en el caso de las restauraciones de las superficies afectadas por incendios forestales donde se deben realizar actuaciones en un breve plazo de tiempo y se deben coordinar las diferentes iniciativas privadas de restauración. La complejidad técnica del proceso restaurador, la inversión económica que precisa y sus consecuencias sobre el conjunto de la sociedad donde se implementa, exige la elaboración de una directriz sobre los trabajos de restauración (Fig.6), con el objetivo de garantizar:

i) La correcta y completa caracterización de los condicionantes del proyecto.

En la fase de diagnóstico del proyecto se deberá hacer referencia de una forma detallada y precisa a los condicionantes impuestos por documentos de planificación, a los condicionantes legales, ecológicos (bióticos, abióticos, perturbaciones, etc.) y socioeconómicos del proyecto, con el detalle preciso para poder identificar y seleccionar las alternativas técnicas más apropiadas. Merece hacerse especial referencia a lo deficiente que son muchos de los estudios sobre el suelo o la inexistente consideración entre los condicionantes al paisaje, el régimen de perturbaciones o los aspectos socio económicos, en muchos de los proyectos de restauración. Los análisis de vulnerabilidad (ALlOZA Y VALLEJo, 2006; VeGA, 2007), pueden ser una herramienta muy interesante para la definición de las acciones prioritarias en los casos de proyectos de restauración que precisen actuaciones de estabilización o de rehabilitación, como es el caso de las superficies afectadas por incendios forestales (GAO, 2006). ii) La definición de los objetivos de la repoblación y la estimación del grado de consecución de los mismos.

Los proyectos de restauración deben responder a unos objetivos concretos y evaluables. En este sentido, la Memoria técnica debe disponer de un epígrafe titulado Evaluación del proyecto en donde se detalle, mediante unos indicadores, la previsión de su grado de consecución, en función de las decisiones elegidas.

iii) La mayor eficiencia técnica, económica y ambiental en las soluciones propuestas.

Todo proyecto debe garantizar la viabilidad técnica, económica y ambiental de las soluciones propuestas a través del proceso de elección de las alternativas que debe garantizar una correcta identificación y evaluación de las mismas. Además, todo proyecto debería disponer de un estudio de impacto ambiental.

iv) La aceptación y participación de la sociedad afectada en el proyecto

La viabilidad de los proyectos de restauración puede verse comprometida por la falta de integración de los mismos en el modelo de gestión desarrollada en ese ámbito rural (LAMB, 2009). Es por ello que hay que facilitar la participación social en el proyecto en su fase de elaboración para poder conocer sus expectativas (LLOVET et al., 2012), consensuar los objetivos del proyecto y garantizar la aceptación de las soluciones técnicas que en él se aporten. Puede, en los casos que se estimen convenientes, valorar la participación en la fase de ejecución, mediante la figura del voluntariado ambiental, con la finalidad de conseguir una mayor identificación y sensibilización con el proyecto de restauración y un modo de canalizar las iniciativas de asociaciones, organizaciones o particulares que pudieran estar interesados en el mismo. En los casos de proyectos de restauración que afecten a número considerable de propietarios forestales debería fomentarse el asociacionismo entre los mismos con objeto de hacer de una manera única a las diferentes actuaciones de la restauración. 
Merece especial referencia el caso de los aprovechamientos maderables en superficies afectadas por incendios forestales donde se precisa concentrar la oferta además de disponer de contratos marco con el sector de la industria para poder agilizar la comercialización de los productos en el caso de que tengan un valor económico (SERRADA, 2013).

v) La disponibilidad de los materiales y técnicas elegidas.

El desarrollo temporal de los proyectos debe permitir la existencia de todos los materiales seleccionados en el mismo y de forma especial de los materiales forestales de reproducción. Para poder adecuar la oferta de planta forestal a las características de la demanda, en cuanto a la categoría de los materiales forestales de reproducción, debe garantizarse al sector viverístico el conocimiento con suficiente antelación de dicha demanda para que pueda satisfacerla. La falta de disponibilidad de estos materiales exige el aplazamiento o suspensión del proyecto de restauración (IgLESIAS et al., 2012). En el caso de superficies afectadas por incendios debe valorarse tener un stock suficiente de semillas de las especies y procedencias ubicadas en áreas con gran frecuencia de incendios con objeto de poder atender la demanda que pudiera surgir para hacer frente a las actuaciones de estabilización o rehabilitación (SERRADA, 2013).

vi) El control de calidad de los trabajos.

Debe generalizarse el establecimiento de planes de control de calidad de la ejecución de los proyectos, que ya se viene realizando por iniciativa de algunas empresas públicas, para poder garantizar que la ejecución se ha realizado conforme a las prescripciones establecidas en el pliego de condiciones (SERRADA et al., 2005). Garantizar la calidad de los trabajos es el punto de partida para poder realizar la evaluación del proyecto e interpretar correctamente los resultados. Existe un antecedente muy interesante, que debe ser valorado en su conjunto, y es el control de calidad integral de la actividad repoblado- ra desarrollado por la Generalitat valenciana (GARCÍA et al., 2008).

vii) El seguimiento del proyecto para evaluar su evolución y adecuación a las previsiones realizadas.

Además de la evaluación a priori, que se incorpora en el proyecto debe, realizarse una evaluación a posteriori donde se estime el grado de cumplimiento real de los objetivos. Para ello deberá establecerse un programa de seguimiento durante los primeros años del proyecto con objeto de poder valorar su evolución, detectando las circunstancias que puedan variar y alterar el diseño previsto. Hay que tener en cuenta que los objetivos del proyecto muchas veces se conseguirán a medio plazo, por lo que el programa de seguimiento deberá tener una duración temporal que permita estimar su grado de consecución. El seguimiento del proyecto permitirá, además, contribuir a evaluar la respuesta de las alternativas desarrolladas que, comparándolas con la respuesta natural del ecosistema, permitirá valorar su grado de acierto (MoreIRA et al., 2012). Pocos proyectos de restauración, en la actualidad, cuentan con programas de seguimiento, por lo que es difícil valorar el éxito del mismo así como extraer conclusiones sobre el resultado obtenido por las diferentes técnicas ensayadas, lo que limita sus posibilidades de mejora. Se han propuesto diferentes recomendaciones, estructuras o directrices para la realización de los planes de seguimiento y evaluación de los proyectos de restauración (NAVARRo-CERrillo et al., 2009; BAUTISTA y Alloza, 2009) que, además, deberían contar con sus respectivos presupuestos para poderlos hacer efectivos.

viii) El análisis espacial y temporal de las repoblaciones realizadas.

La creación de un banco o base de datos de todos los proyectos de repoblación, financiados con dinero público, precisa de ser implementada por la necesidad de disponer de una información territorial amplia que permita futuros análisis, de 


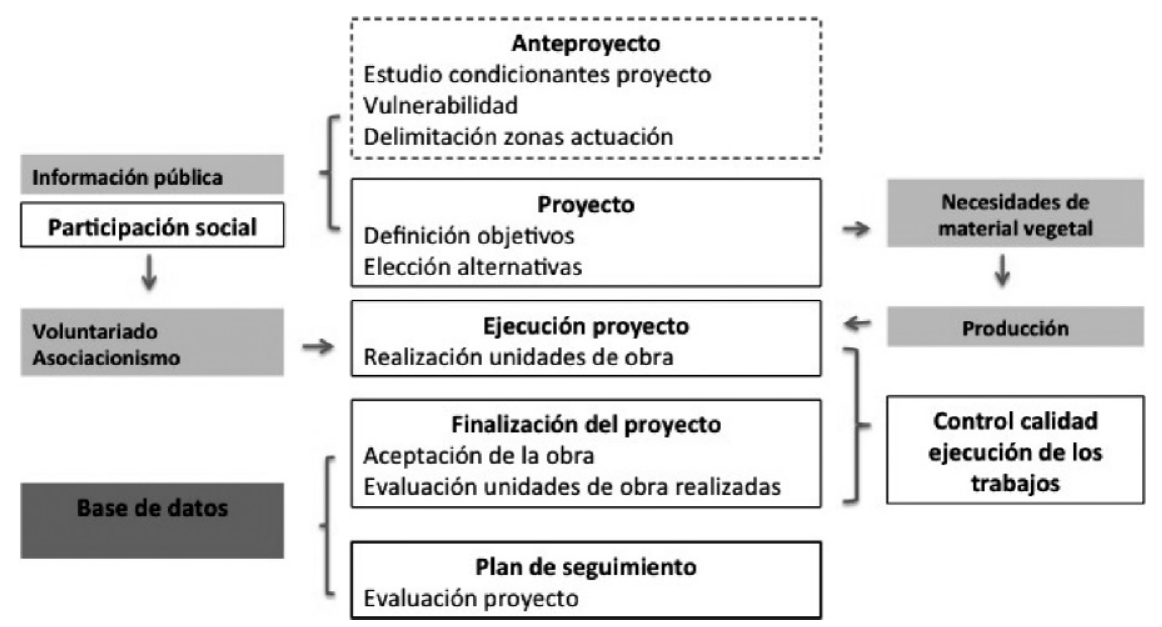

Figura 6. Esquema de las fases más relevantes del proceso restaurador

carácter temporal o espacial, a largo plazo. Esta base de datos deberá contener, además de la información cartográfica, la información más relevante del proyecto y del plan de seguimiento.

\section{BIBLIOGRAFÍA}

ASEMFO, Foresdat. www.foresdat.es.

AllozA, J. \& VAllejo, R.; 2006. Restoration of burned areas in forest management plans. In: W. Kepner, J. Rubio, Da Mouat \& F. Pedrazzini (eds.), Desertification in the Mediterranean Region: 475-488. Springer. Heidelberg.

ANDRÉS, C. \& OJEDA, F.; 2002. Effects of afforestation with Pinus pinaster on biodiversity of Mediterranean heathlands in South Spain. Biodivers. Conserv. 11: 1511-1520.

Ariza, D.; Navarro-Cerrillo, R.; Del Campo, A.; IBAÑEZ, A. Y JORRÍN, J.; 2008. Influencia de la fecha de plantación al establecimiento de Pinus halepensis. Aplicación de la proteómica a estudios de ecofisiología de campo. Cuad. Soc. Esp. Cienc. For. 28: 111-117.

Brooker, R.W.; Maestre, F.T.; Callaway, R.M.; Lortie, C.L.; CAVIERES, L.A.; Kunstler, G.; Liancourt, P.; Tielbörger, K.; Travis, J.M.; Anthelme, F.; Armas, C.; Coll, L.; Corcket, E.; Delzon, S.; Forey,
E.; Kikvidze, Z.; Olofsson, J.; Pugnaire, F.; Quiroz, C.L.; SaCcone, P.; Schiffers, K.; Seifan, M.; Touzard, B. \& Michalet, R.; 2008. Facilitation in plant communities: the past, the present, and the future. J. Ecol. 96: 18-34.

BAI, W.; Zhang, H.; LiU, B.; Wu, Y. \& Song, J.; 2010. Effects of super-absorbent polymers on the physical and chemical properties of soil following different wetting and drying cycles. Soil Use Manage. 26: 253-260.

Bautista, S. \& AllozA, J.; 2009. Evaluation of forest restoration projects. In: S. Bautista, J. Aronson \& V. Vallejo (eds.), Land restoration to combat desertificatio: 47-72. CEAM. Valencia.

BurdetT, A.; 1990. Physiological processes in plantation establishment and the development of specifications for forest planting stock. Can. J. For. Res. 20: 415-427.

Callaway, R. \& Walker, L.; 1997. Competition and facilitation: a synthetic approach to interactions in plant communities. Ecology 78: 1958-1965.

CARRERAS, C.; 2006. Diversificación estructural de masas forestales artificiales. Resultados de ensayos en Andalucía Oriental. Invest. Agrar:: Sist. Rec. For: 103-110.

PARdos CARrión, J.A.; 1988. Mejora genética de especies arbóreas forestales. Fundación Conde del Valle de Salazar. Madrid. 
CASTRO, J.; ZAMORA, R.; HódAR, J.; GÓmeZ, J. \& GÓMEZ-APARICIO, L.; 2002. Use shrubs as nurse plants for restoration in Mediterranean mountains: a 4-year study. Rest. Ecol. 10: 297-305.

Clemente, A.; Werner, C.; Máguas, C.; Cabral, M.; Martins-LouçaO, M. \& CORREIRA, O.; 2004. Restoration of a limestone quarry: effect of soil amendments on the establishment of native mediterranean sclerophyllous shrubs. Rest. Ecol. 12: 20-28.

CORCHERO, S.; GoZAlO, M.; VILlar-SAlVADOR, P. \& PeÑuelas, J.; 2002. Crecimiento radical en campo de Pinus halepensis y Quercus ilex plantados en diferentes momentos. Montes 5-11.

Cortina, J.; Peñuelas, J.; PuÉrtolas, J.; Savé, R. Y Vilagrosa, A.; 2006. Calidad de la planta forestal para la restauración en ambientes mediterráneos. Estado actual de los conocimientos. Organismo Autónomo de Parques Nacionales. Madrid.

Del Campo, A.; Navarro-Cerrillo, R.; AguileLLA, A. Y FLORS, J., 2008. Influencia microclimática del diseño del tubo protector y respuesta de diez especies forestales al tubo ventilado. Cuad. Soc. Esp. Cienc. For. 28: 81-87.

Del Campo, A.D.; Hermoso, J.; Flors, J.; Lidon, A. \& NAVARro-CERrillo, R.M.; 2011. Nursery location and potassium enrichment in Aleppo pine stock 2. Performance under real and hydrogel-mediated drought conditions. Forestry 84: 235-245

DAVIS, S. \& JACOBS, D.F.; 2005. Quantifying root system quality of nursery seedlings and relationship to outplanting performance. New For. 30: 295-311.

ElORRIETA, J.; 1946. Importancia de las razas forestales en las repoblaciones. Montes 9: 198-205.

FAO; 2010. Evaluación recursos forestales mundiales. FAO 2010. Roma.

$\mathrm{GAO} ; 2006$. Wildland fire rehabilitation and restauration. U.S. Government Accountability Office.

Gachet, S.; Leduc, A.; Bergeron, Y.; NGUYenXUAN, T. \& TREMBLAY, F.; 2007. Understory vegetation of boreal tree plantations: Differences in relation to previous land use and natural forests. Forest Ecol. Manage. 242: 49-57.
García, A.; Mena, J.; Moreno, M.; García, R.; Molina, S.; Albert, J. \& TÁrraga, R.; 2008. Control integral de calidad de repoblaciones forestales: planteamiento y primeros resultados en la provincia de Valencia. Cuad. Soc. Esp. Cienc. For. 28: 119-124.

GrossNiCKLE, S.C.; 2012. Why seedlings survive: influence of plant attributes. New For. 43 711-738.

GóMEZ, J.; 2003. Spatial patterns in long distance dispersal of Quercus ilex acorns by jays in a heterogeneous landscape. Ecography 26: 573-584.

GÓMEZ-APARICIO, L.; ZAMORA, R.; GÓMEZ, J.; HódAR, J.; CASTRO, J. \& BARAZA, E.; 2004. Applying plant facilitation to forest restoration: a meta-analysis of the use of shrubs as nurse plants. Ecol. Appl. 14: 1128-1138.

Gómez-Aparicio, L.; Zavala, M. \& Bonet, F.; 2009. Are pine plantations valid tools for restoring Mediterranean forests? An assessment along abiotic and biotic gradients. Ecol. Appl. 19: 2124-2141.

GonzÁlez-Moreno, P.; Quero, J.L.; PoOrTer, L.; BONET, F.J. \& ZAMORA, R.; 2011. Is spatial structure the key to promote plant diversity in Mediterranean forest plantations? Basic Appl. Ecol. 12: 251-259.

GuTIÉRREZ-RAVÉ, J.; 2007. Respuesta hídrica de suelos semiáridos marginales sometidos a distintos tratamientos para repoblación forestal. Tesis doctoral. Universidad de Granada.

HAASE, D.; 2011. Seedling root targets. USDA Forest Proceedings, 80-82.

HERNÁNDEZ, L.: 2013. Estándares para la certificación de proyectos de restauración de ecosistemas forestales. En: Actas VI Reunión Grupo Repoblaciones Forestales de la S.E.C.F.: 7-11. Sociedad Española de Ciencias Forestales. Madrid.

HütTermann, A.; Zommorodi, M. \& ReISE, K.; 1999. Addition of hydrogels to soil for prolonging the survival of Pinus halepensis seedlings subjected to drought. Soil Till. Res.: 295-304.

Iglesias, S.; Nicolás, J.; Alía, R.; Peñuelas, J.; Prada, M.; Juan, A.S.; Calvo, J.; Rueda, E.; Mallofret, E.; Pérez, V.; SAMANiego, B.; VAllejo, M.; Martín, E.; Villanueva, I. \& FernándeZ, G.; 2012. 
Protocolo técnico a aplicar en lo relativo al material forestal de reproducción en la redacción y ejecución de proyectos de repoblación y restauración forestal. Organismo Autónomo Parques Nacionales.Madrid.

LAMB, D.; 2009. Economic, social and cultural factors affecting landscape restoration. $I n$ : S. Bautista, J. Aronson \& V. Vallejo (eds.), Land restoration to combat desertification: 35-46. CEAM. Valencia.

Llovet, J.; Alloza, J.; Bautista, S.; Guixot, L.; Mayor, A.; Murias, C.; Vilagrosa, A. \& Vallejo, V.; 2012. Proyecto PRACTICE. Evaluación de alternativas de gestión frente a la desertificación incorporando la participación y experiencias locales. Flamma 4: 51-60.

Lugo, A.; 1997. The apparent paradox of reestablishing species richness on degraded lands with tree monocultures. Forest Ecol. Manage. 99: 9-19.

Magrama; 2014. Anuarios Estadística Forestal. [En línea] Disponible en: www.magrama.gob.es/biodiversidad/estadisticas/forestal_anuarios_todos.aspx.

Margolis, H. \& BRAND, D.; 1990. An ecophysiological basis for understanding plantation establishment. Can. J. For. Res. 20: 375-390.

Martínez De Azagra, A.M. \& Del Río, J.; 2012. Los riegos de apoyo y de socorro en repoblaciones forestales. Foresta: 32-54.

Mongil, J. \& Martínez De Azagra, A.; 2006. Técnicas de recolección de agua y de oasificación para el desarrollo de la agricultura y la restauración forestal en regiones desfavorecidas. Cuad. Geogr. 40: 67-80.

Montero, G.; Cisneros, O.; Cañellas, I.; HeRnÁNDEZ, A.; FríAs, M. Y VAllejo, R.; 2002. Manual de selvicultura para plantaciones de especies productoras de madera de calidad. Ediciones Mundi-Prensa. Madrid.

Moreira, F.; Arianoutsou, M.; Vallejo, V. \& HERAS, J.; 2012. Setting the scene for post-fire management. In: F. Moreira, M. Arianoutsou, P. Corona \& J. de las Heras (eds.), Post-Fire Management and Restoration of Southern European Forests: 1-20. Springer. Heidelberg.

NAVARro-CERrillo, R.; Fragueiro, B.; Ceaceros, C.; Del Campo, A. \& De Prado, R.; 2005. Establishment of Quercus ilex L. subsp. ballota [Desf.] Samp. using different weed control strategies in southern Spain. Ecol. Eng. 25: 332-342.

NAVARro-Cerrillo, R.; Bautista, S.; GuzMÁN, J.; Herrera, R.; Lara, P.; Torres, M.; Ceacero, C. \& Del Campo, A.; 2009. Monitoring guidelines for the implementation of forest restoration projects in Mediterranean regions. In: S. Bautista, J. Aronson \& V. Vallejo (eds.), Land restoration to combat desertification: 73-86. CEAM. Valencia.

NiCOLÁs, J. Y Iglesias, S.; 2012. Normativa de comercialización de los materiales forestales de reproducción. En: J. Pemán, R. NavarroCerrillo, J.L. Nicolas y R. Serrada (eds.), Producción y Manejo de semillas y plantas forestales I: 3-32. Organismo Autónomo de Parques Nacionales. Serie Forestal. Madrid.

Palacios, G.; Cerrillo, R.M.; Del Campo, A. \& TORAL, M.; 2009. Site preparation, stock quality and planting date effect on early establishment of Holm oak (Quercus ilex L.) seedlings. Ecol. Eng. 35: 38-46.

Pausas, J.; Bladé, C.; Valdecantos, A.; Seva, J.; Fuentes, D.; Alloza, J.; Vilagrosa, A.; Bautista, S.; Cortina, J. \& Vallejo, R.; 2004. Pines and oaks in the restoration of Mediterranean landscapes of Spain: new perspectives for an old practice-a review. Plant Ecol. 171: 209-220.

Pemán, J. \& VAdell, E.; 2009. Reconstrucción de la estadística de la actividad repobladora desde 1879 hasta nuestros días. En: S.E.C.F.-Junta de Castilla y León (eds.), Actas 5 Congreso Forestal Español 5CFE01-669: 1-14. Sociedad Española de Ciencias Forestales. Pontevedra.

Pemán, J.; Peguero-Pina, J.; Valladares, F. \& Gil-Pelegrín, E.; 2010. Evaluation of unventilated treeshelters in the context of Mediterranean climate: Insights from a study on Quercus faginea seedlings assessed with a 3D architectural plant model. Ecol. Eng. 36: 517-526.

Pemán, J.; Latorre, B. \& Ocaña, L.; 2011. Use of Pinus halepensis in restoration of semiarid burned area of the Ebro Valley. In: IUFRO Conference on Restoring Forests: Advances in Techniques and Theory.

Pemán, J.; NAVARro-Cerrillo, R.; Nicolás, J.; Prada, M. \& Serrada, R. (eds.); 2012. 
Producción y manejo de semillas y plantas forestales. I. Organismo Autónomo de Parques Nacionales. Serie Forestal. Madrid.

Pons, J. \& Pausas, J.; 2007. Acorn dispersal estimated by radio-tracking. Oecologia 153: 1432-1939.

Prevosto, B.; Monnier, Y.; Ripert, C. \& FERnÁnDEZ, C.; 2011. Diversification of Pinus halepensis forests by sowing Quercus ilex and Quercus pubescens acorns: testing the effects of different vegetation and soil treatments. Eur. J. For. Res. 130(2): 67-76.

Puerta-Pinero, C.; Gómez, J.M. \& Schupp, E.W.; 2010. Spatial patterns of acorn dispersal by rodents: do acorn crop size and ungulate presence matter? Oikos 119: 179-187.

Puértolas, J.; Oliet, J.A.; Jacobs, D.F.; Benito, L.F. \& PEÑUelas, J.L.; 2010. Is light the key factor for success of tube shelters in forest restoration plantings under Mediterranean climates? Forest Ecol. Manage. 260: 610-617.

Querejeta, J.; Roldán, A.; Albaladejo, J. \& CAstillo, V.; 2001. Soil water availability improved by site preparation in a Pinus halepensis afforestation under semiarid climate. Forest Ecol. Manage. 149: 115-128

RADOGLOU, K.; RAFTOYANNIS, Y. \& Halivopoulus, G.; 2003. The effect of planting date and seedling quality on field performance of Castanea sativa and Quercus frainetto. Forestry 5: 569-578.

REES, M.; 2013. Competition on productivity gradients - what do we expect?. Ecol. Lett. 16: 291-298.

Reyna, S. \& Colinas, C.; 2007. Truficultura. En: S. Reyna (ed.), Truficultura. Fundamentos y técnicas: 237-275. Mundi Prensa. Madrid.

Reyna, S.; De Miguel, A.; Palazón, C.; HonruBIA, M.; Ágreda, T.; Águeda, B.; GarcíABARREDA, S.; MARTÍn, M.; MorenO-ARroyo, B.; OliaCh, D.; SÁNCHEZ, S. \& BARRIUSO, J.; 2013. Spanish trufficulture. En: Actas I Congreso Internacional de Truficultura. Teruel.

RITCHIE G.A.; 1984. Assessing seedling quality. In: M.I. Dureya \& T.D.. Landis (eds.), Forest nursery manual: Production of bareroot seedlings. Martinus Nijhoff/Dr W. Junk Publishers. Research Laboratory, Oregon State University. Corvallis.
Ruiz De La Torre, J.; 1993. Objetivos de diversidad biológica en la reforestación de tierras agrícolas. Elección de especie y densidad de implantación. Montes 34: 20-30.

Sánchez, J.; Ortega, R.; Hervás, M.; Padilla, F. Y PUIGNAIRE, F.; 2004. El microrriego, una técnica de restauración de la cubierta vegetal para ambientes semiáridos. Cuad. Soc. Esp. Cienc. For. 17: 109-112.

SECF, 2010. Situación de los bosques y del sector forestal en España. Informe 2010. Sociedad Española de Ciencias Forestales. Madrid.

Serrada, R.; Navarro-Cerrillo, R. y Pemán, J.; 2005. La calidad de las repoblaciones forestales: una aproximación desde la selvicultura y la ecofisiología. Inv. Agrar.: Sist. Rec. For. 14: 462-481.

SERRADA, R.; 2013. Restauración de masas de Pinus pinaster afectadas por incendios forestales. Lleida.

South, D.; Rose, R. \& Mcnabb, K.; 2001. Nursery and site preparation interaction research in the United States. New For. 22: 43-58.

Torroba, P.; Zaldívar, M.; FERnándeZSAntos, B. y MartíneZ-Ruíz, C.; 2013. El papel de los matorrales autóctonos en la expansión del bosque en minas de carbón restauradas en el norte de Palencia. En: Actas de la VI Reunión del Grupo de Repoblaciones de la SECF: 81-89.

Valladares, F.; Balaguer, L. Y Mola, I.; 2011. Restauración ecológica e infraestructuras de transporte: definiciones, problemas, desafíos. En: F. Valladares, I. Balaguer, I. Mola, A. Escudero y V. Alfaya (eds.): Restauración ecológica de áreas afectadas por infraestructuras de transporte: 16-39. Ministerio de Medio Ambiente Rural y Marino. Madrid

Valle, G.; SARdÁ, P.; Aguilar, A.; Villar-SalVADOR, P. Y PEÑUELAS, J.; 2005. Crecimiento radical de plantones de Pinus halepensis y $\mathrm{P}$. pinea durante el periodo húmedo del año. $E n$ : S.E.C.F. (ed.): Actas 4 Congreso Forestal Español 4CFE05-129: 1-7. Sociedad Española de Ciencias Forrestales. Zaragoza.

VEGA, J.; 2007. Bases ecológicas para la restauración preventiva de zonas quemadas. En: Conferencia Internacional sobre Incendios Forestales. Sevilla. 
Villar-Salvador, P.; Puértolas, J.; Cuesta, B.; PeÑuelas, J.; UsCola, M.; Heredia-GuerreRO, N. \& REY-BENAYAS, J.; 2012. Increase in size and nitrogen concentration enhances seedling survival in Mediterranean plantations. Insights from an ecophysiological conceptual model of plant survival. New For. 43: 755-770.

VÁzQuez, A.; Oliet, J. y Pú́rtolas, J.; 2013. Efecto de la transmisividad del tubo protector y la sequía sobre el establecimiento de dos especies mediterráneas Quercus ilex y
Pinus halepensis. En: Actas de la VI Reunión del Grupo de Repoblaciones de la SECF: 101-106.

Willoughby, I.; Balandier, P.; Bentsen, N.S.; McCarthy, N. \& Claridge, J.; 2009. Forest vegetation management in Europe. European Science Foundation.

ZAMORA, R.; HódAr, J. \& MATías, L.; 2010. Positive adjacency effects mediated by seed disperser birds in pine plantations. Ecol. Appl. 20: 1053-1060. 